\title{
OÙ EN EST L'AMÉLIORATION GÉNÉTIQUUE DE L'OKOUMÉ AU GABON?
}

\author{
F. GRISON
}

L'Okoumé (Aucoumea klaineana Pierre, Burséracée) est à plusieurs titres la principale essence forestière du Gabon :

- c'est une essence très abondante en forêt de basse altitude, qui se régénère très bien sur les terrains dégagés, en particulier sur les anciennes cultures : elle est donc d'autant plus abon. dante dans une région, que l'homme y a été plus actif, et il existe de nombreux peuplements purs d'Okoumés en forêt naturelle, qui ont été l'objet d'importants travaux de sylviculture entre 1936 et 1960 ;

- l'Okoumé est, depuis plusieurs décennies, grâce à sa bonne aptitude à la fabrication de contreplaqué la principale essence exploitée au Gabon, malgré la présence de nombreuses autres essences de valeur, exploitées en moindres quantités. Les premières exportations de grumes d'Okoumé datent de $1892(7)^{*}$;

- c'est pratiquement la seule espèce plantée par les services chargés du reboisement (les premières plantations datent de 1935 ; en 1970, environ 26000 ha avaient été plantés) et c'est l'espèce qui a été la plus étudiée par les recherches forestières du Gabon.

L'action du service forestier en faveur de la forêt à Okoumés a été multiple : entre 1930 et 1939 , des "réserves d'aménagement " ont été créées, dans lesquelles on souhaitait protéger les peuplements de jeunes okoumés. Après la guerre, les travaux d'amélioration des peuplements natureis se sont intensifiés et ont été poursuivis jusqu'en 1960. A partir de 1935 et, surtout, de 1944, des essais de plantations ont permis la mise au point progressive d'une bonne méthode de sylviculture de l'Okoumé. Depuis 1960, les travaux d'amélioration des peuplements naturels ont été délaissés au profit des plantations ärtificielles (10).

Les recherches se sont intéressées principalement à la sylviculture de l'Okoumé, à la conservation de ses graines, à l'amélioration de l'état sanitaire des pépinières et des plantations. L'abondance des graines que l'on peut facilement ramasser en forêt ou sur les routes, au moment de leur chute en février, était telle qu'on n'avait jamais eu à se préoccuper de leur production (il suffisait de savoir les conserver un an pour faire face aux années de mauvaise récolte). Cependant, la régénération de la forêt par plantations à grande échelle permettait d'envisager, et justifiait, des travaux d'amélioration génétique de l'Okoumé.

(1)* Les chiffres entre parenthèses renvoient à la bıbliographie. 
Une tournée de M. P. Bouvarel en 1966 a permis de jeter les bases d'un programme d'amélioration génétique (6) qui a commencé bien sûr, par un essai de provenances. Celui-ci fut mis en place en 1967 dans la réserve de la M'VOUM. Des essais de greffage et de bouturage, en 1969-70 puis en 1972, ont donné des résultats encourageants, mais ils ont été interrompus par le départ de MM. Bedel puis Grouazel, qui les avaient entrepris. Enfin le Centre technique torestier tropical a créé en décembre 1972 une division d'amélioration génétique des arbres forestiers dont le but était, tout simplement, de créer des vergers à graines de semences sélectionnées pour le service des reboisements.

\section{PROGRAMME DES TRAVAUX}

Il était facile de prévoir que l'amélioration génétique de l'Okoumé passerait par des sélections, des essais de croisements, et peut-être aussi du bouturage.

Il fallait donc commencer par étudier sa variabilité génétique, afin de savoir si une sélection serait possible et à quel niveau elle serait la plus efficace : provenances, descendances, individus. En outre, on pourrait ainsi connaître les meilleures sources de graines.

Les études de reproduction végétative devraient être poursuivies et développées : greffage et marcottage pour les besoins de la recherche, bouturage pour ceux du reboiseur.

La connaissance de la reproduction sexuée de l'Okoumé permettrait de réaliser des croisements et d'implanter des vergers à graines.

Enfin, on pouvait s'interroger sur l'héritabilité de certains caractères qu'on serait tenté de sélec. tionner.

On voit que ce programme de travaux visait l'acquisition de connaissances et la mise au point de techniques sur lesquelles se fonderait ensuite le programme d'amélioration génétique. Il s'agissait, en fait, de travaux préliminaires. Ce sont eux qui nous ont occupé pendant plus de quatre ans, et dont nous donnons ici les résultats (4).

\section{ÉTUDE DE LA VARIABILITÉ GÉNÉTIQUE}

Une première récolte de graines a permis la mise en place en 1967, dans la réserve de la M'VOUM, d'une plantation comparative de treize provenances représentant l'aire de l'Okoumé dans son extension nord-sud, de la Guinée Équatoriale au Congo. Cette plantation, en six répétitions, couvre 26 ha. Les graines ont été semées en poquets. Des taux de germination trés variables selon les provenances (les plus proches ont le mieux germé), des problèmes d'hétérogénéité du sol à l'intérieur des blocs, des dégâts dus aux chutes des branches d'arbres empoisonnés, rendent impossible une comparaison fine entre les provenances. II ressort néanmoins de cet essai que les différences de hauteurs totales entre les provenances sont peu importantes, et qu'elles tendent à s'atténuer avec l'àge.

De toutes les mesures prises : hauteur totale, nombre de branches, nombre de folioles des deux plus jeunes feuilles, angle formé par la tige avec les deux plus jeunes feuilles, deux diamètres perpendiculaires à mi-distance de la deuxième et de la troisième feuille, l'analyse révéle que les deux premières sont les plus discriminantes. 


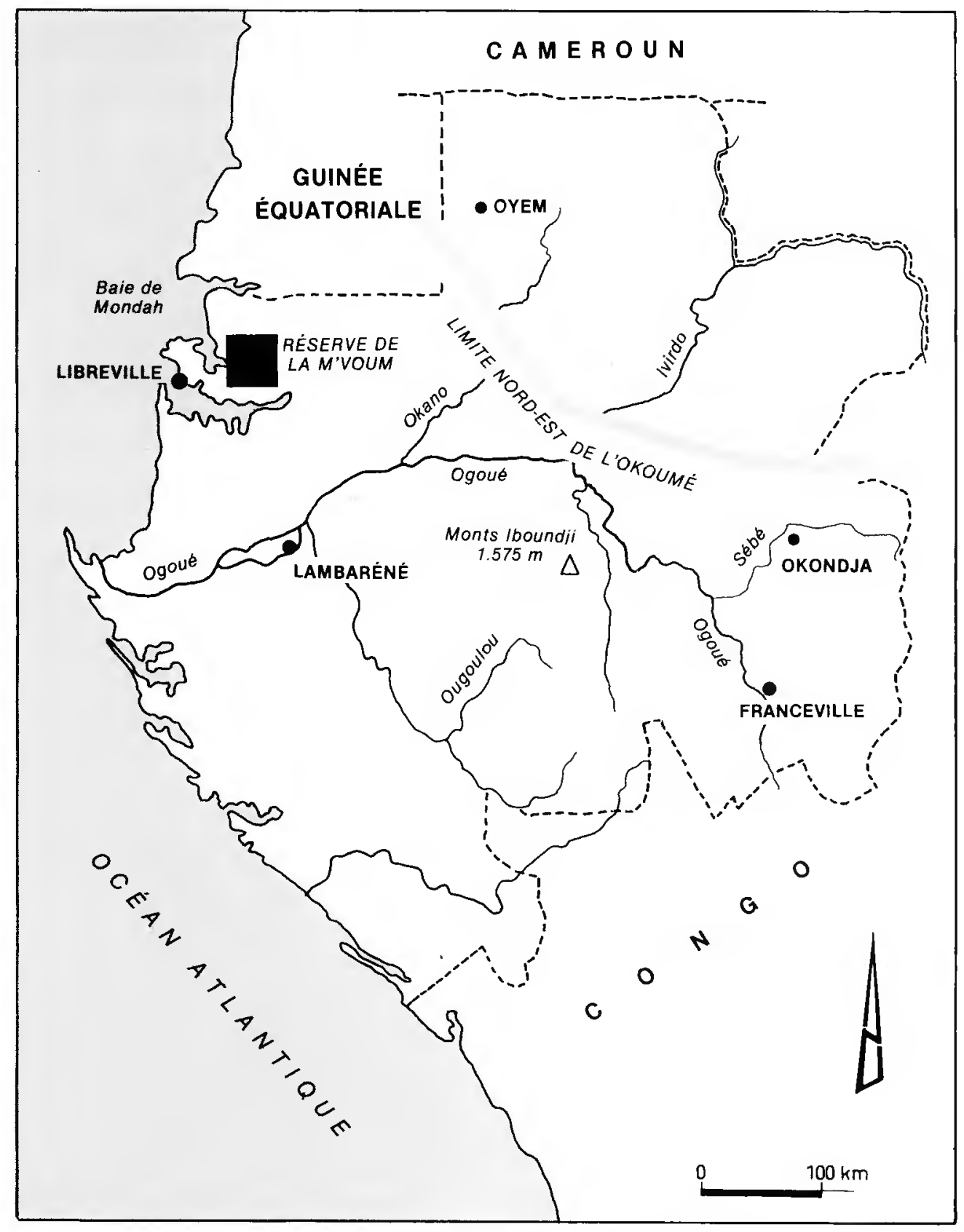

L'Okoumé se rencontre dans tout le Gabon à l'exception de la partie nord-est du pays. II semble que cette limite coïncide avec la limite climatique. Au nord de cette limite, le ralentissement hivernal des pluies devient une véritable petite saison sèche. Or, c'est durant cette période que la fructification a lieu. Les graines amenées par le vent des régions voisines tombent sur un sol sec et, leur pouvoir germinatif étant de courte durée, il est possible qu'elles le perdent avant le retour des pluies. (Extrait de "La torét au Gabon " par G. de SAINT-AUBIN - 1963.) 
Une deuxième récolte de graines, en 1974, a été réalisée directement par les chercheurs du Centre technique torestier tropical - Gabon," afin de s'assurer des conditions de récolte constantes pour toutes les provenances. Les graines ont été récoltées sous un nombre de semenciers aussi élevé que possible, sans privilégier les okoumés les plus prolifiques. Les provenances ont été choisies pour leur situation dans les milieux les plus divers possible : du sud au nord (saison sèche de plus en plus courte, pluviosité croissante); du niveau de la mer à l'altitude la plus élevée (mais nous n'avons pas trouvé de graines sous les okoumés du Mont Iboundji, point culminant du Gabon); de l'ouest à l'extrême est (Okondja) de l'aire naturelle de l'Okoumé. En outre, vingt trois descendances ont été récoltées dans trois provenances, sous des semenciers pris au hasard.

Cette récolte a permis la réalisation de deux plantations comparatives : I'une à M'Viadi (réserve de la M'VOUM) en 1975, l'autre en forêt de la Mondah en 1976.

La plantation de $M$ 'Viadi couvrait 3,5 ha et contenait trois répétitions de dix provenances et deux répétitions de vingt trois descendances. Afin d'éviter les dégâts dus aux chutes des branches, nous avons entiérement déforesté et dégagé le terrain à planter, à l'aide d'un bouteur et de scies à chaîne ; mais les passages répétés de l'engin sur un sol que les premières pluies commençaient à mouiller, ont décapé irrégulièrement la mince couche humifere, et la lenteur de l'installation du recrû entre les lignes d'Okoumé a nui à la croissance initiale de ces derniers. Mais cet essai a donné quelques résultats : les provenances nord et est (Oyem et Okondja) sont les moins bonnes ; les provenances de la Mondah sont les meilleures. Les différences entre descendances sont très importantes, y compris entre descendances d'une même provenance.

Cependant l'abondance des éléphants était telle dans ce secteur, que les villageois eux-mêmes, las des dévastations causées à leurs cultures vivrières, envisageaient de s'établir ailleurs. Notre plantation, qui était un îlot de recrû et de jeunes okoumés en zone de haute forèt, était particulièrement attrayante pour ces animaux. S'ils n'ont pas supprimé la plantation, les dégâts qu'ils ont causés à partir de 1976 ont tout au moins détruit son caractére de plantation comparative.

La plantation de la Mondah comportait quatre répétitions de dix provenances sur 2,3 ha, et sept répétitions de vingt descendances sur 2,2 ha. Cette plantation, située près de Libreville, ne risquait pas d'être visitée par les éléphants. En outre, elle bénéficiait de l'expérience acquise l'année précédente à M'Viadi. Le terrain, préparé par l'École nationale des eaux et forêts, était bien dégagé mais pas décapé. Le principal défaut de cette plantation était d'être jeune au moment de notre départ du Gabon. Mais comme elle était particulièrement bien venue, certains résultats provisoires pouvaient déjà en être tirés 13 mois aprés la plantation. Comme à M'Viadi, les provenances Oyem et Okondja sont les moins bonnes, et celles de la Mondah (et Mbel) sont les meilleures. Il est heureux que ce soit généralement à la Mondah que soient récoltées les graines d'Okoumé utilisées dans les reboisements. De grandes différences apparaissent entre les descendances où l'on peut cependant distinguer trois groupes, avec en tête deux provenances de la Mondah et une d'Oyem, et en dernière position trois descendances d'Oyem.

Après mai 1977 cet essai n'aurait plus été entretenu. Pourtant au prix d'un effort minime d'entretien pendant deux ou trois ans, cet essai aurait pu nous apprendre encore beaucoup.

Ces essais de provenances, trop jeunes ou trop imparfaits, ne permettent pas de comparaisons fines entre les lots. Mais il s'en dégage nettement que des différences entre provenances peuvent être exploitées. Pour des planfations dans l'Estuaire, la Mondah semble être la meilleure

\footnotetext{
" Qu'il me soit permis d'adresser mes vifs remerciements à H.F. Maître, qui m'aida beaucoup pendant cette récolte.
} 


\section{F. GRISON}

provenance. Des différences importantes existent entre les descendances, et d'excellentes descendances peuvent être récoltées ailleurs que dans la Mondah, ce qui permet d'envisager d'intéressants essais de croisements entre individus de provenances différentes.

\section{ÉTUDE DE LA REPRODUCTION VÉGÉTATIVE}

Le greffage de l'Okoumé se fait " en bouteille ". Les premières greffes réussies ont été faites par cette méthode à Loudima (Congo) en 1970 (11). Au Gabon en 1972, M. Grouazel, après avoir essayé sans succès plusieurs méthodes de greffage, réussissait lui aussi des greffes en bouteille. C'est cette méthode que nous avons cherché à préciser en réalisant plus de 1200 greffes en bouteille.

Nous préconisons le greffage, en août-septembre, de greffons avec ou sans bourgeon terminal, ayant 6 à $9 \mathrm{~mm}$ de diamètre au niveau de la ligature et $40 \mathrm{~cm}$ de long environ, dont $15 \mathrm{~cm}$ audessus de l'incision, $15 \mathrm{~cm}$ au niveau de l'incision (inciser jusqu'au tiers du diamètre), $10 \mathrm{~cm}$ en dessous pour que le bas du greffon plonge dans l'eau de la bouteille. Le pourcentage de reprise est connu trois mois après le greffage (2).

Cette technique permet l'implantation de parcs à clones et de vergers à graines. Le taux de reprise obtenu, de l'ordre de $40 \%$ en greffant sur des sauvageons, devrait pouvoir être amélioré par une équipe permanente et entraînée de pépiniéristes utilisant des porte-grettes homogènes cultivés spécialement (ou mieux, bouturés).

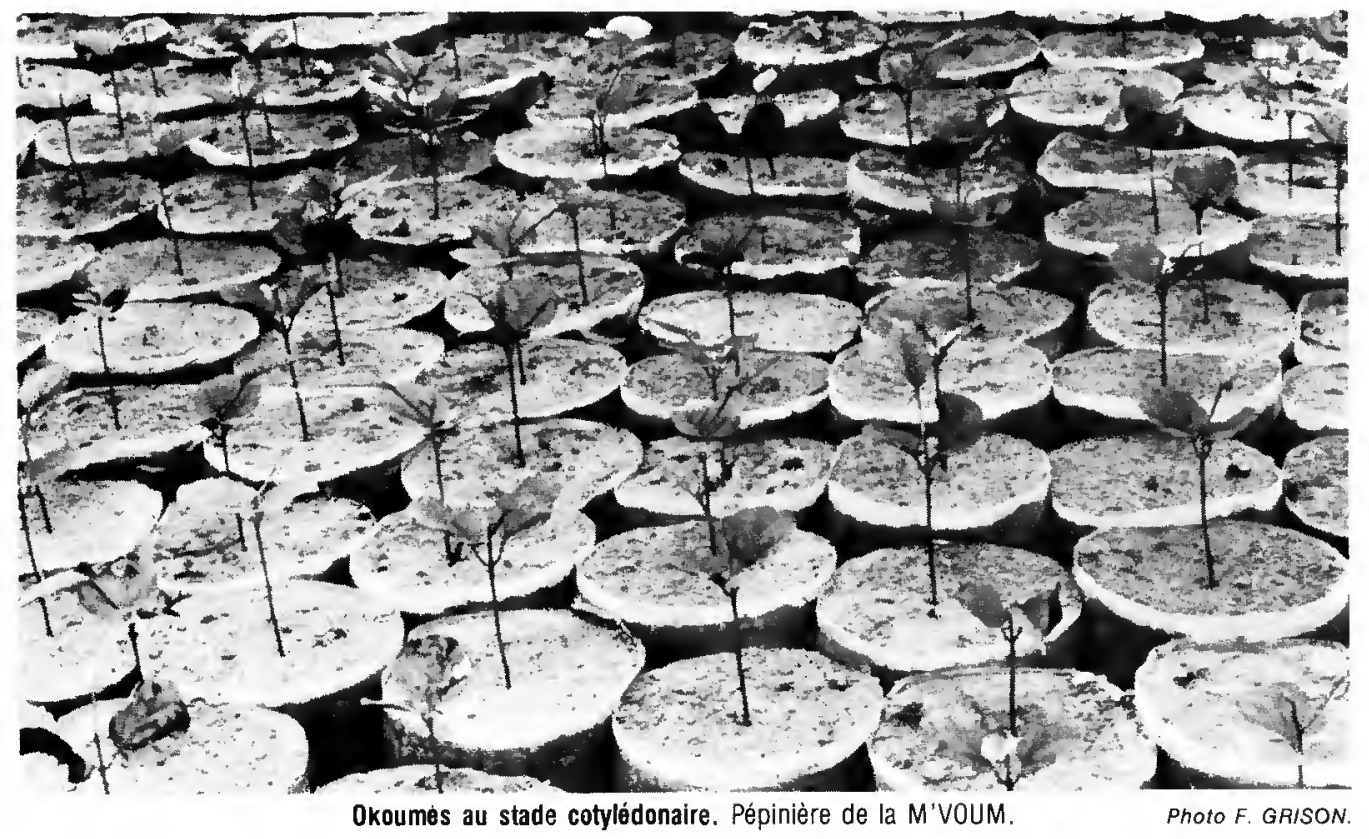


Le bouturage de tiges adultes avait donné quelques résultats en 1970 avec J. Bedel (11). Mais les succès remportés par le Centre technique forestier tropical au Congo dans le bouturage herbacé, d'Eucalyptus principalement, mais aussi de Limba et d'Okoumé (12), nous ont incité à étudier cette méthode au Gabon en 1975, en reprenant et en essayant de transposer aux conditions de Libreville, les résultats obtenus au Congo. C'est M.-O. Hamel qui fut chargé de ces essais. II disposait d'une installation de brouillard artificiel qu'on laissait en permanence pendant la journée et qui était interrompue la nuit. En dépit de fréquentes coupures d'eau et de pannes de son installation, M.-O. Hamel a obtenu des résultats très encourageants.

Le substrat le meilleur pour les boutures paraît être le sachet contenant dans sa moitié supérieure du sable dans lequel se forment les racines, et dans sa moitié inférieure de la terre humifère dans laquelle les racines pénètrent ensuite, ce qui simplifie le sevrage. Les boutures issues de rejets d'arcure, de rameaux d'arbres adultes, de plants greffés ont échoué. Celles issues de jeunes plants ont donné quelques reprises. Les boutures de rejets ont le mieux réussi. Les rejets doivent être jeunes, fins (diametre : $4 \mathrm{~mm}$ ), à tige jaune - marron. Ces boutures ont repris à $45 \%(9 / 20)$ en moins de deux mois (5).

Les travaux de bouturage doivent maintenant ètre développés à grande échelle par une bonne équipe de pépinière.

Le marcottage aérien a été l'objet d'un essai sur des perches de $6 \mathrm{~m}$ de haut. Les marcottes sur tige intacte ont échoué. II y a eu une émission de racines (7/10) sur celles dont la tige avait été écorcée sur un anneau de $3 \mathrm{~cm}$ de haut, ce qui montre qu'il est possible de marcotter l'Okoumé.

Ces résultats montrent que la reproduction végétative de l'Okoumé est possible. Nous considérons qu'ils sont suffisants pour que le greffage et le bouturage sortent du domaine purement expérimental. Leur mise au point devra être poursuivie par les utilisateurs.

\section{ÉTUDE DE LA REPRODUCTION SEXUÉE}

Cette étude devait répondre, en vue de l'amélioration génétique de l'Okoumé, à deux questions fondamentales :

- Quel est le régime de reproduction de l'Okoumé (auto- ou allogamie) ?

- Comment réaliser des croisements contrôlés ?

En fait, elle nous réservait une surprise majeure car, contrairement à ce que l'on pensait jusqu'à présent, l'Okoumé est une essence dioïque dont seule la fleur mâle était connue et interprétée comme une fleur hermaphrodite. Le pistillode qui se trouve en son centre est avorté et n'évolue jamais en fruit (8). La première question était donc réglée de la façon la plus simple : dioïque, l'Okoumé est obligatoirement allogame.

Une tour de 20, puis $22 \mathrm{~m}$ de haut, dressée dans un taillis d'okoumés de $12 \cdot 13$ ans sur souches de 24 - 25 ans permettait d'observer in situ les fleurs, qui sont groupées en grappes à l'extrémité des rameaux. L'emballage de grappes, ou de parties de grappes, dans des sacs à pollinisation ou dans des cages de grillage, le comptage fréquent des boutons, fleurs, fruits de trois grappes, la mesure pendant toute leur existence de la longueur et du diamètre de 59 fruits, la réalisation d'essais de pollinisation manuelle, nous ont conduit aux conclusions suivantes $(3,9)$ : 


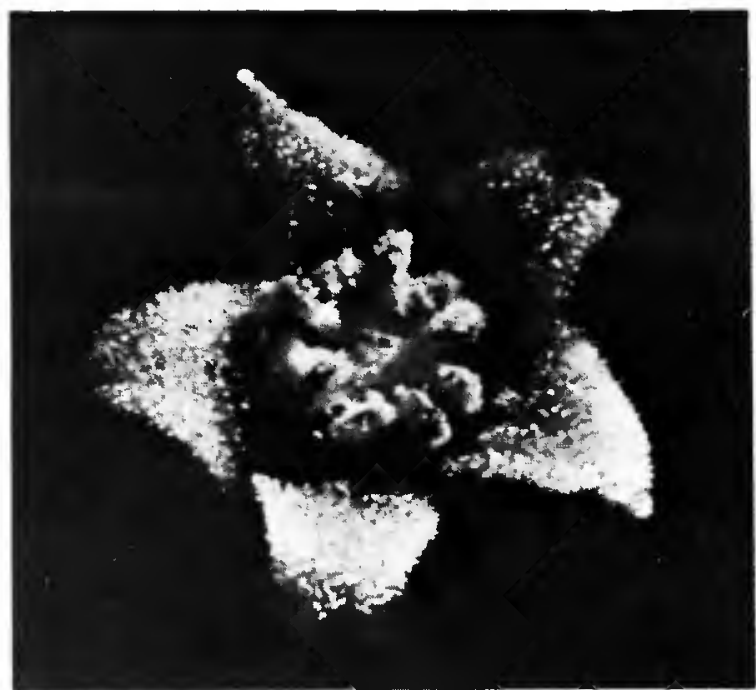

Fleur mâle.

Le pollen reste adhérent aux étamines.

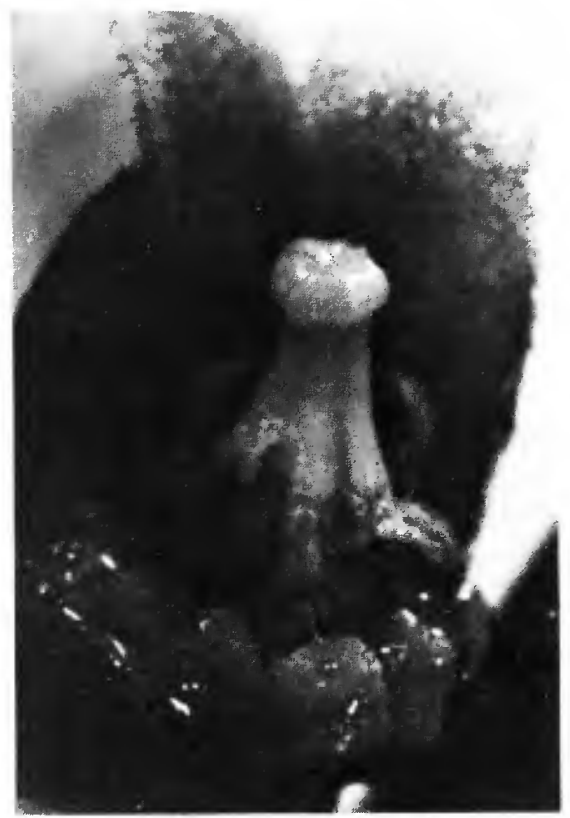

Fleur femelle.

3 pétales et 3 sépales enlevés.

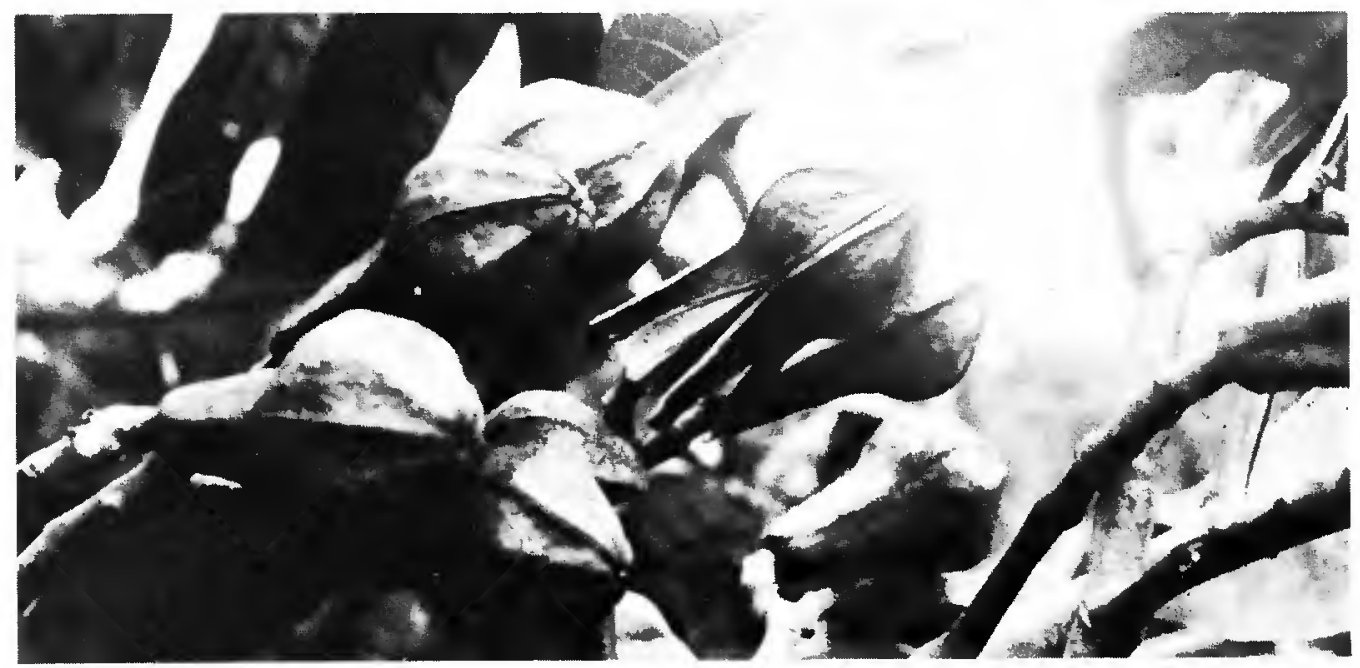

Déhiscence des fruits (drupe déhiscente à 5 noyaux ailés). Photos F. GRISON.

- la pollinisation est réalisée en septembre-octobre par des insectes. Les fleurs femelles s'épanouissent lentement et demeurent réceptives pendant plusieurs jours. Quelques jours après la pollinisation, le périanthe tombe, le fruit rougit puis verdit. Sa déhiscence intervient 80 jours après la pollinisation ;

- les grappes supportent très bien les sacs à pollinisation. Pour réaliser une pollinisation manuelle, il faut mettre en sac une grappe ne portant pas de fleurs épanouies. Une semaine plus tard, on pollinise les fleurs épanouies, par contact direct avec des fleurs mâles (nous n'avons fait 
aucune manipulation de pollen), puis on remet le sac. Une semaine plus tard, on retire le sac et on ne laisse sur la grappe que les jeunes fruits, issus de la pollinisation manuelle.

Les principaux objectifs que visait cette étude ont été atteints, puisque on sait maintenant que l'Okoumé est allogame, que l'on connaît le déroulement de sa floraison, et qu'une méthode simple de pollinisation manuelle donne d'excellents rẻsultats.

\section{CONCLUSION}

L'Okoumé est l'essence la plus intéressante économiquement pour le Gabon et sa sylviculture a fait l'objet de nombreux travaux dont certains déjà anciens. Son amélioration génétique ne fait que commencer et on mesure combien les connaissances de base peuvent être encore fragmentaires...

Cependant, un véritable programme d'amélioration génétique peut maintenant être entrepris.

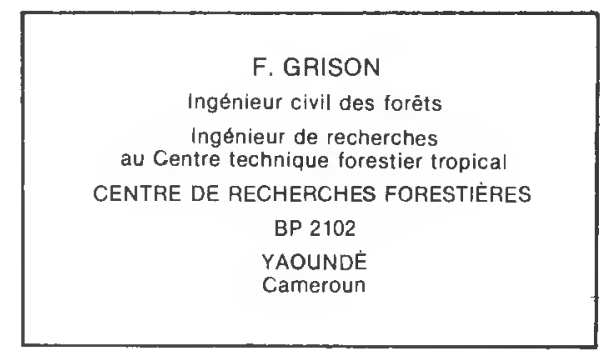

\section{BIBLIOGRAPHIE}

Pour plus d'informations sur les travaux effectués, on peut consulter au Centre technique forestier tropical (45 bis, avenue de la Belle-Gabrielle, 94130 Nogent-sur-Marne) les rapports suivants :

(1) BEDEL (J.). - Premiers résultats des essais de bouturage de l'Okoumé réalisés au Gabon, - 1970.

(2) GRISON (F.). - Greffage de l'Okoumé. Résultats des essais entrepris au Gabon de 1969 à 1974. - 1976.

(3) GRISON (F.). - Etude de la biologie florale de l'Okoumé. - 1977.

(4) GRISON (F.). - Amélioration génétique de l'Okoumé. Etat des connaissances et des travaux au 31 mars 1977. - 1977.

(5) HAMEL (O.). - Bouturage de l'Okoumé. Résultats des essais entrepris au Gabon en 1975. - 1976.

Citons également :

(6) BOUVAREL (P.). - Amélioration génétique de l'Okoumé. Etude préliminaire et projet de programme de travail. - 1966.

(7) FORÉT. - Okoumé ou bois du Gabon (Note dactylographiée). - 1970.

(8) GRISON (F.). - Note sur les fleurs de l'Okoumé (Aucoumea klaineana Pierre, Burséracée). Adansonia, ser. 2, vol. 17, $n^{\circ} 3,1978$, pp. 335-342.

(9) GRISON (F.). - Amélioration génétique de l'Okoumé. Bois et forèts des Tropiques, $n^{\circ} 178$ et $179,1978$.

(10) LEROY-DEVAL (J.). - Biologie et sylviculture de l'Okoumé. Tome 1 : La sylviculture de l'Okoumé. Nogent-sur-Marne, Ed. du Centre technique forestier tropical, 1976.

(11) MARTIN (B.), QUILLET (G.). - Premiéres greffes de Limba et d'Okoumé. - 1970.

(12) MARTIN (B.), QUILLET (G.). - Bouturage des arbres forestiers au Congo. Bois et forêts des Tropiques, $n^{\circ} 154$ à 157,1974 . 